\title{
MULTI-EPOCH, DUAL-FREQUENCY VLBI OBSERVATIONS OF PKS 1830-211 FROM JAPAN
}

\author{
YOSHIAKI HAGIWARA \\ The Graduate University for Advanced Studies, \\ Nobeyama Radio Observatory, Nagano 384-13, Japan \\ K. FUJISAWA, P. EDWARDS, H. HIRABAYASHI, Y. MURATA ANL \\ H. KOBAYASHI \\ The Institute of Space and Astronautical Science, \\ 3-1-1 Yoshinodai, Sagamihara, Kanagawa 229, Japan \\ AND \\ T. IWATA \\ Communications Research Laboratory, \\ Kashima Space Research Center, Ibaraki 314, Japan
}

\section{Observations}

PKS 1830-211 is a strong, flat-spectrum compact double source with a component spacing of 1 arcsecond. Observations of PKS 1830-211 were made with the Japanese domestic VLBI network at $2.3 \mathrm{GHz}$ and $8.4 \mathrm{GHz}$ bands in sessions between December 1991 and November 1994. The Usuda $64 \mathrm{~m}$ (ISAS) and Kashima $34 \mathrm{~m}$ (CRL) telescopes were used for all observations, and were used in conjunction with the Mizusawa $10 \mathrm{~m}$ (NAO) for observations in 1994. In addition, the total flux was measured with the Usuda $64 \mathrm{~m}$ at both bands. Data was recorded using K3 and K4 formatters and recorders, and correlated with NAOCO (the New Advanced One-unit COrrelator of the National Astronomical Observatory).

\section{Analysis and Results}

The model fitting results presented here were obtained from one $2 \mathrm{MHz}$ bandwidth channel of the Usuda-Kashima baseline data. Results from other VLBI experiments enable us to assume values for the position angle and 
TABLE 1. $2.3 \mathrm{GHz}$ observations and Usuda-Kashima baseline results. Errors in fluxes are estimated to be of the order of $10 \%$.

\begin{tabular}{lcccc}
\hline Date & Length [hr] & Total flux [Jy] & Corr. flux [Jy] & Flux ratio \\
\hline 1991-Dec-16 & 5.5 & 9.1 & 2.2 & $1.15 \pm 0.06$ \\
1992-Mar-25/26 & 5.5 & 8.9 & 4.1 & $1.10 \pm 0.06$ \\
1992-Jun-18 & 7 & 8.7 & 3.9 & $1.12 \pm 0.06$ \\
1994-Mar-25/26 & 2 & 10.1 & 5.6 & $1.18 \pm 0.02$ \\
1994-Nov-4/5 & 2.5 & - & - & $1.08 \pm 0.02$ \\
\hline
\end{tabular}

component separation and thus to determine the flux ratio of the two components. The flux ratios show little variation in either band over 3 years but the visibilities show distinct variations during this period. The $2.3 \mathrm{GHz}$ results are given in Table 1; similar results were obtained at $8.4 \mathrm{GHz}$. This indicates that the time-scale for the increase in the correlated flux density of both components is less than the separation between our observations.

\section{Discussion}

The $2.3 \mathrm{GHz}$ correlated flux on the Usuda-Kashima baseline is a significantly smaller fraction of the total flux than at $8.4 \mathrm{GHz}$. This indicates that much of the broad ring feature, which contributes to the total flux at $2.3 \mathrm{GHz}$, is resolved out on this baseline (130 mas resolution at $2.3 \mathrm{GHz})$. The flatter spectrum core is thus more dominant at $8.4 \mathrm{GHz}$. In addition, the low galactic latitude and longitude of the source result in the source being increasingly broadened at lower frequencies by the effects of interstellar scintillation, which reinforces the expectation of higher correlated fluxes at higher frequencies (see also Jones et al. 1995).

The synchronization of the flux variation of both components is consistent with the effects of gravitational lensing. Recently, van Ommen et al. (1995) have derived a propagation delay of $44 \pm 9$ days for one component with respect to the other. Our observations were not designed for time resolution, so we are not able to observe this effect. Regular monitoring at radio wavelengths will enable the time delay to be constrained more tightly.

\section{References}

Fujisawa, K., 1992, M. Sc. Thesis, Tokyo University (unpublished).

Hagiwara, Y., 1995, M. Sc. Thesis, Nagoya University (unpublished).

Jones, D., et al., 1995, these proceedings.

van Ommen, T.D. et al., 1995, ApJ, 444, 561. 